\title{
Neoliberalismo y medio ambiente: una aproximación desde la geografía crítica
}

\author{
Hug March \\ Universitat Oberta de Catalunya \\ hug.march@gmail.com
}

Recepción: enero de 2012

Aceptación: marzo de 2012

\section{Resumen}

En este estado de la cuestión, reviso la literatura sobre la neoliberalización del medio ambiente para arrojar luz sobre los procesos que lo convierten en una nueva esfera de acumulación y circulación de capital. La privatización y la mercantilización son los dos procesos clave mediante los cuales se articula el proyecto neoliberal. Más allá de éstos, la desregulación o la rerregulación, así como la comercialización o la corporatización merecen ser singularizadas. La geografía crítica parte de una posición privilegiada para entender estos procesos y analizar sus consecuencias sobre el mundo humano y no humano. A su vez, puede y debe plantear alternativas válidas a los discursos y a las prácticas hegemónicas de gestión ambiental neoliberal: la modernización ecológica y el ambientalismo de mercado.

Palabras clave: neoliberalismo; medio ambiente; geografía crítica; ambientalismo de mercado; privatización; mercantilitzación.

\section{Resum. Neoliberalisme i medi ambient: una aproximació des de la geografia critica}

En aquest estat de la qüestió, reviso la literatura sobre la neoliberalització del medi ambient, amb l'objectiu d'aclarir els processos que el converteixen en una nova esfera d'acumulació i circulació de capital. La privatització i la mercantilització són dos dels processos clau mitjançant els quals s'articula el projecte neoliberal. Més enllà d'aquests, la desregulació o la reregulació, així com la comercialització o la corporatització mereixen ser singularitzades. La geografia crítica parteix d'una posició avantatjada per entendre aquests processos i analitzar-ne les conseqüències sobre el món humà i no humà. $\mathrm{Al}$ mateix temps, pot plantejar alternatives vàlides als discursos i a les pràctiques hegemòniques de gestió ambiental neoliberal: la modernització ecològica i l'ambientalisme de mercat.

Paraules clau: neoliberalisme; medi ambient; geografia crítica; ambientalisme de mercat; privatització; mercantilització.

\section{Résumé. Néolibéralisme et environnement: une approche depuis la géographie critique}

Cet article fait une révision de la littérature sur la néoliberalisation de l'environnement. L'objectif principal est mettre en lumière les multiples processus qui visent à convertir l'environnement en une nouvelle sphère de circulation et accumulation de capital. La 
privatisation ainsi que la marchandisation sont deux processus clé du projet néolibéral. Toutefois, d'autres processus parmi lesquels la dérégulation, la rérégulation, la commercialisation ou même la corporatisation doivent être singularisées. La géographie critique est dans une position optime pour comprendre ces processus ainsi que pour analyser leurs conséquences sur le monde humain et non-humain. Au même temps il peut et doit proposer des alternatives valides au discours et pratiques hégémoniques concernant la gestion de l'environnement: la modernisation ecologique et l'environnementalisme de marché.

Mots clé: néolibéralisme; environnement; géographie critique; environnementalisme de marché; privatisation; marchandisation.

\begin{abstract}
Neoliberalism and the Environment: An Approach from Critical Geography
In this state-of-the-art review, I examine the literature on the neoliberalization of the environment. The main objective is to shed light on the multiple processes that turn the environment into a new sphere for the accumulation and circulation of capital. Privatization and commodification are key processes that vertebrate the neoliberal project. However, other processes such as deregulation, reregulation, as well as commercialization or corporatization should be singled out. Critical geography is in a good position to understand these processes and analyze their consequences on the human and non-human world. At the same time, valid alternatives to the hegemonic discourses and practices of neoliberal environmental management, namely ecological modernization and market environmentalism, can and should be proposed by critical geography.
\end{abstract}

Keywords: neoliberalism; environment; critical geography; market environmentalism; privatization; commodification.

\title{
Sumario
}

1. Introducción 5. Conclusiones

2. Neoliberalismo y pensamiento 6. Agradecimientos

geográfico Bibliografía

3. Neoliberalismo y medio ambiente

4. Ambientalismo de mercado y modernización ecológica: paradigmas dominantes en la gestión del medio ambiente a comienzos del siglo XXI

\section{Introducción}

Entrado el siglo Xxi, el medio ambiente se ha convertido en un reto para los sectores productivos. Y no sólo en un reto, sino también en una oportunidad de negocio. Aunque en el imaginario colectivo la protección del medio ambiente aún se contempla como un escollo para el desarrollo económico, también se observa una tendencia a la incorporación del medio ambiente en los circuitos internacionales de circulación y acumulación de capital.

Para contrarrestar los beneficios decrecientes en las actividades industriales tradicionales y los problemas de sobreacumulación, el capital privado se 
reinventa y busca nuevas oportunidades una vez la expansión geográfica y las soluciones espaciales (spatial fix) teorizadas por Harvey $(1982,2003)$ parecen agotadas. La tendencia espontánea del mercado a expandirse (Carvalho y Rodrigues, 2006) se traduce en la búsqueda incesante de nuevas esferas de acumulación de capital. El medio ambiente no escapa de esta dinámica: las soluciones ambientales (environmental fixes) a los problemas de sobreacumulación del capital (Castree, 2008a, 2008b) constituyen un elemento importante en el sistema económico vigente.

Históricamente, el capitalismo ha dependido de los recursos naturales en tanto que materias primas para el funcionamiento y la expansión de los procesos industriales y de urbanización. Entrado el siglo xxi, observamos como esferas de la naturaleza que estaban fuera del mercado son incorporadas en las lógicas de acumulación capitalista, principalmente a través del establecimiento de nuevos derechos de propiedad y de lógicas mercantiles. Ejemplos de esto los encontramos en la creación del mercado europeo de emisiones de dióxido de carbono, la creación de mercados del agua, la monetización de servicios ambientales o los derechos de propiedad sobre materiales genéticos. Noel Castree (2008a) argumenta que la relación entre el modelo neoliberal y el medio ambiente es paradójica, ya que, a través de la lógica de acumulación de capital, se busca proteger y degradar el mundo biofísico, a la vez que se producen «nuevas» naturalezas, como, por ejemplo, los organismos modificados genéticamente, que son incorporadas en los circuitos nacionales e internacionales del capital. Todo lo anterior ocurre en un contexto de dominio ideológico de lo que conocemos como neoliberalismo (Self, 1993; Toke, 2000), en el que los distintos modelos capitalistas convergen hacia prácticas y discursos hegemónicos (Coates, 2000).

\section{Neoliberalismo y pensamiento geográfico}

El neoliberalismo, como sostienen Larner et al. (2007), ha ido reemplazando a la globalización como sujeto de análisis en muchas disciplinas de las ciencias sociales, entre las cuales encontramos la geografía. Así pues, el neoliberalismo y los procesos de neoliberalización (England y Ward, 2007) se han convertido en un término básico para entender las tendencias político-económicas entrado el siglo XXI (Block, 2001).

Definir qué es el neoliberalismo no es una tarea fácil (England y Ward, 2007). En el sentido más general, por neoliberalismo entendemos la filosofía económica y política que cuestiona (e incluso rechaza) la intervención del gobierno en los mercados y en las relaciones económicas de los individuos (Heynen et al., 2007a). Noel Castree (2008a) conceptualiza el neoliberalismo no simplemente como una filosofía económica o una práctica hegemónica, sino como un modelo de gobernanza exhaustivo que pretende naturalizar el mercado como el medio para evaluar y distribuir las necesidades y los lujos de la vida.

Este paradigma nació a mediados del siglo xx de la mano de economistas como Friedrich Von Hayek (2000 [1944]) o Milton Friedman (1962) y se 
desplegó en la década de 1970 cuestionando el consenso keynesiano vigente (Tickell y Peck, 2003; Peck, 2004) en el marco de estancamiento económico y crisis del estado fordista, especialmente en Estados Unidos e Inglaterra. David Harvey (2005) argumenta que la emergencia de este paradigma está estrechamente vinculada a la restauración del poder de clase y de la lógica de acumulación del capital. Sin embargo, Leitner et al. (2007) defienden que la emergencia y la supremacía de este paradigma no se debe a su superioridad intelectual ni a los mecanismos de clase expuestos por Harvey. Contrariamente, estos autores afirman que su triunfo se debió al hecho que era atractivo para distintos agentes opuestos al fordismo y al keynesianismo: los neoconservadores vieron en él una oportunidad para promover la libertad y la responsabilidad individual; los capitalistas lo vieron como una oportunidad para incrementar sus beneficios, al disminuirse la intervención del estado, $\mathrm{y}$, finalmente, el estado lo vio como una oportunidad para afrontar su crisis fiscal. En este sentido, Bob Jessop (2002) conceptualiza el neoliberalismo como el modo dominante de regulación postkeynesiana.

Independientemente de su conceptualización como ideología o modo de gobernanza, el neoliberalismo ha ido evolucionando desde su despliegue en la década de 1970 como reacción al keynesianismo. En este sentido, Tickell y Peck (2003) hablan de tres fases distintas en su desarrollo: el protoneoliberalismo (década de 1970), el roll-back Neoliberalism (es decir, aquel que pretendía reducir las funciones del estado para restaurar el statu quo prekeynesiano) y, finalmente, el roll-out Neoliberalism.

Esta última fase, vigente desde la década de 1990 y con el Consenso de Washington de 1994 como elemento clave, se puede definir como el periodo en el que la ideología neoliberal ha impregnado y se ha afianzado de manera hegemónica en los discursos político-económicos y ha condicionado las arquitecturas de gran parte de las instituciones internacionales y de los gobiernos nacionales (Tickell y Peck, 2003). Para categorizar esta hegemonía, Crotty (2000) llama «régimen neoliberal global» a la fase actual del capitalismo. Su hegemonía a nivel discursivo determina el «qué» puede ser debatido y el «cómo» (Carrier, 1997). La esfera del debate se reduce a conceptos tecnocráticos y la confrontación ideológica o el disenso son substituidos por la cooptación de los discursos disidentes. Al reducirse el espectro de alternativas posibles, Tickell y Peck (2003) hablan de la despolitización del debate público y Erik Swyngedouw (BAVO, 2007) plantea directamente la condición postpolítica. En un sentido focauldiano, el neoliberalismo se basa en el "gobierno en la distancia» (Larner, 2000; Larner et al., 2007; Latour, 2005; Miller y Rose, 2008) y llega a impregnar al individuo con una «subjetividad neoliberal» (Leitner et al., 2007).

La crisis económica desatada con la caída de Lehman Brothers en 2008, que, en un principio, parecía hacer tambalear la ideología neoliberal, ha servido, contrariamente, para afianzar su supremacía, con lo cual ha demostrado su resiliencia y su capacidad de mutación e hibridación. El neoliberalismo, según Peck y Tickell (2002), parece estar presente en todos los sitios y es mostrado como el sentido común de nuestros tiempos. 
Sin embargo, es importante resaltar que este modo de gobernanza llega a distintos lugares también de manera distinta (Gamble, 2006) en función de las coreografías de poder existentes (Beeson, 2007). A su vez, se articula y se hibridiza con otros proyectos políticos y cobra distintas formas materiales (Larner, 2003; Larner et al., 2007; Lockie y Higgins, 2007; Peck, 2004; Perreault y Martin, 2005), a veces contradictorias, que pueden dar lugar a resultados inesperados. Consecuentemente, y sin olvidar su clara génesis, tenemos que diferenciar entre las teorizaciones sobre el neoliberalismo y el «neoliberalismo realmente existente» (actually existing Neoliberalism, Brenner y Theodore, 2002a) y recordar que el capitalismo oscila entre procesos de diferenciación o desarrollo desigual, tal y como nos recuerda Neil Smith (1984), y procesos de homogeneización (Robertson, 2007). Así pues, existen diversas geografías del neoliberalismo, tanto en un sentido espacial como teórico.

Después de esta concisa introducción al neoliberalismo y a los procesos de neoliberalización desde una perspectiva eminentemente geográfica, a continuación se revisa la literatura geográfica sobre los procesos de neoliberalización del medio ambiente.

\section{Neoliberalismo y medio ambiente}

Si bien a finales del siglo xx y comienzos del siglo xxI el nexo entre medio ambiente y neoliberalismo no fue muy examinado (McCarthy y Prudham, 2004), en los últimos años, ha ido apareciendo un importante número de trabajos sobre el tema, especialmente desde la geografía crítica anglosajona (Castree, 2010). El libro Neoliberal Environments: false promises and unnatural consequences (Medios ambientes neoliberales: promesas falsas y consecuencias no naturales) (Heynen et al., 2007a) es, probablemente a día de hoy, el exponente más claro de este esfuerzo. Este libro se centra en el análisis de las dinámicas y los complejos e imprevisibles resultados de las intervenciones neoliberales en el medio ambiente. Sin embargo, y más allá de analizar los impactos ambientales de estas reformas, el libro se interesa en analizar cómo lo que conocemos como movimiento ecologista realza la emergencia del proyecto neoliberal e incluso coincide y se articula con él (Heynen et al., 2007b). Así, si, por un lado, el medio ambiente puede ser movilizado discursivamente para mostrar los límites y cuestionar el modelo económico hegemónico, por otro lado, la ortodoxia neoliberal puede circular e hibridizar con el ecologismo o el ambientalismo.

David Harvey (1996) afirma que todos los proyectos socioecológicos son proyectos políticos y viceversa. En este sentido, la evolución de lo que conocemos por gobernanza ambiental es coconstitutiva de la evolución del proyecto neoliberal (Heynen et al., 2007a). No obstante, como recalca Noel Castree (2006), la neoliberalización del medio ambiente presenta un «desconcertante carácter amorfo».

Para entender mejor la interrelación entre neoliberalismo y medio ambiente, es importante distinguir los distintos procesos a través de los cuales se 
despliega la neoliberalización del medio ambiente: la privatización, la comercialización, la corporatización, la desregulación/rerregulación y la mercantilización.

\subsection{La privatización de la naturaleza}

La privatización es un momento clave en el proceso de neoliberalización del medio ambiente; a través de ella, se materializa el proyecto neoliberal. En este sentido, resulta imprescindible abordar este proceso para entender la reorganización contemporánea de las relaciones entre naturaleza y sociedad bajo el manto del modelo neoliberal hegemónico (Liverman, 2004; Mansfield, 2007a, 2008).

Becky Mansfield (2008) argumenta que la privatización se inserta en un proceso más general que limita el acceso a los recursos a través del cercamiento (enclosure), mediante el cual a estos recursos se les asigna propiedad y pueden ser controlados y transferidos. Esta autora describe tres procesos básicos de privatización. El primero es el cercamiento de bienes comunales (commons), que tiene como lógica básica la «acumulación primitiva» descrita por Karl Marx (1975) o la más reciente «acumulación por desposesión» planteada por David Harvey (2003). Esta primera categoría incluiría procesos de privatización del agua (Bakker, 2005, 2010; Swyngedouw, 2005), de las tierras (Wolford, 2007), de la flora y la fauna salvajes (Moore, 2011; Robbins y Luginbuhl, 2007), de los minerales (Bridge, 2007) o de las pesquerías (Mansfield, 2001, 2004, 2007b; St. Martin, 2007), entre otros ejemplos. Una segunda categoría incluiría los procesos que imponen mecanismos de apropiación y derechos de propiedad intelectual a esferas de la vida que habían permanecido fuera de las lógicas económicas, como el cuerpo humano o, a escala menor, el material genético (Prudham, 2008), incluso los procesos y los componentes bioquímicos y biológicos. La creación de un mercado de dióxido de carbono a nivel europeo entra en esta categoría (Bailey y Maresh, 2009). En esta misma línea, Noel Castree (2008a) describe el proceso de privatización como la asignación de derechos claros de propiedad sobre fenómenos ambientales o sociales que previamente eran controlados por el estado o la comunidad, o simplemente no eran controlados por nadie.

Finalmente, encontramos una tercera tendencia que incluye la privatización de la gestión ambiental mediante la implementación de mecanismos de mercado. Este último tipo de privatización está ligada al dominio del «ambientalismo de mercado" (Market Environmentalism) como paradigma de gestión ambiental dominante en las sociedades occidentales avanzadas (veremos este punto de manera más detallada más adelante).

El estado adquiere un rol central en estos procesos de privatización (Swyngedouw, 2005), en un primer momento, para organizar e iniciar el proceso y, posteriormente, en el seguimiento y el establecimiento de marcos postregulatorios, como Karl Polanyi ya había argumentado en la década de 1940 (2001 [1944]). En otras palabras, como señala Becky Mansfield (2007a, 2008), la privatización no es un proceso natural y evolutivo que ocurra de manera espon- 
tánea, sino que tiene que ser refrendada por el estado mediante la creación de derechos de propiedad. Esta fase de privatización se incluiría en el roll-out Neoliberalism que hemos evocado anteriormente.

\section{2. ¿Regulación, desregulación o rerregulación?}

What does it mean to carefully delimit privatization as separate from deregulation when we can also talk about privatization as a form of regulation? (Mansfield, 2007a: 4)

En la cita expuesta más arriba, Becky Mansfield se pregunta si es necesario separar los procesos de privatización de los procesos de regulación o desregulación de la gestión ambiental o si es mejor considerar la privatización como una forma de regulación.

La desregulación, es decir, la eliminación de barreras (normas, leyes, etc.) para facilitar la expansión desenfrenada del capital, tiende a aparecer en el imaginario colectivo como el eje central del capitalismo contemporáneo. Sin embargo, si partimos de la obra de Karl Polanyi, The Great Transformation (2001 [1944]), podemos ver que el laissez-faire está planeado y que la rerregulación es un proceso igual de relevante que la desregulación para entender los procesos actuales de neoliberalización. La rerregulación se basa en la intervención del estado para facilitar los procesos de privatización y la creación de mercados que controlen aspectos cada vez más amplios de la vida social y ambiental (Castree, 2008a), y reescribe las relaciones y los derechos de las personas y del medio ambiente (Bakker, 2007a). Paradójicamente, en un contexto en que se aboga por la teórica no intervención en la economía, los estados pueden ser muy intervencionistas (Peck, 2004). En este sentido, el economista Joseph Stiglitz (2001 [1944]) argumenta que el «libre mercado» no ha sido nunca del todo libre ni autorregulado, incluso ha llegado a tener un componente utópico (Block, 2001 [1944]). El capitalismo contemporáneo está lleno de «impurezas» (Carvalho y Rodrigues, 2006) y la práctica del neoliberalismo (the practice of Neoliberalism) se basa en la deconstrucción y reconstrucción de instituciones en el nombre del «mercado» (Tickell y Peck, 2003).

\subsection{Comercialización de la naturaleza y corporatización de la gestión pública}

Este proceso implica la reconfiguración de las instituciones de gestión y de toma de decisiones (entendiendo institución en el sentido sociológico: normas, leyes, reglas y costumbres) mediante la introducción de principios (por ejemplo: eficiencia), métodos (por ejemplo: análisis de coste y beneficio) y objetivos comerciales (por ejemplo: maximización de beneficios) (Bakker, 2003, 2005, 2007a; Castree, 2008a ${ }^{1}$. La comercialización reescribe el recurso como un bien

1. Castree habla de marketization en lugar de commercialization, pero podemos asimilar los dos términos. 
económico en lugar de un bien público y redefine a los usuarios como clientes individuales en lugar de ciudadanos (Bakker, 2007b).

En este contexto, los incentivos monetarios tienen un papel central para influenciar el comportamiento individual. Carvalho y Rodrigues (2006) señalan que una dependencia excesiva de los mecanismos monetarios puede ir en detrimento de las prácticas sociales que no pasan por el mercado.

No se tiene que confundir la comercialización con la privatización del medio ambiente. Mientras la primera implica cambios institucionales en la gestión del recurso, la segunda implica cambios organizacionales. Por ejemplo, en el caso del agua, observamos empresas públicas que gestionan el recurso siguiendo principios comerciales y empresas privadas que ofrecen subsidios cruzados a los usuarios con menos recursos, con lo cual se alejan de principios comerciales estrictos.

En cambio, sí que podemos realizar vínculos entre la privatización y la corporatización (corporatization). Este último proceso pretende que el sector público sea más eficiente mediante la emulación de prácticas del sector privado (Smith, 2004), lo que es conocido como «nueva gestión pública» (New Public Management) (Finger y Allouche, 2002; Gamble, 2006; Nickson y Franceys, 2003). Aunque muchos autores desvinculan esta estrategia de la privatización y argumentan que solamente aumenta la eficiencia de los servicios públicos (Smith, 2004²), otros autores establecen vínculos entre estos procesos. En este sentido, la corporatización puede ser un aspecto precursor de la privatización, ya que muchas instituciones internacionales, como el Banco Mundial, lo recomiendan como un paso previo (Bakker y Cameron, 2002; Bakker, 2003). Por otro lado, Esteban Castro (2009) sugiere que esta nueva gestión pública o corporatización ya es, de hecho, el resultado de la inercia de los procesos de privatización. En este sentido, y refiriéndose al agua, argumenta que las estrategias de privatización han inducido a realizar cambios discursivos y conceptuales en la manera de pensar los servicios públicos: los servicios de agua ya no son vistos como un derecho ciudadano, sino que, progresivamente, la gestión pública persigue la generación de beneficios en lugar de un servicio público sostenible bajo control ciudadano.

Más allá de los vínculos con la privatización, lo que sí podemos afirmar es que los procesos de comercialización y corporatización se incluyen dentro del llamado roll-out Neoliberalism (Peck y Tickell, 2002), ya que el estado no desaparece, sino que, por el contrario, adopta valores y mecanismos del sector privado, lo que Noel Castree (2008a) llama market proxies. De este modo, el proyecto neoliberal no significa la muerte del estado, sino la reinvención de su rol y de sus funciones a favor de las lógicas de acumulación de capital (Peck, 2004).

2. Laila Smith (2004), sin embargo, reconoce que los efectos y los impactos de la corporatización pueden ser similares a los de la privatización, ya que se prioriza la eficiencia por encima de la equidad. 


\subsection{Mercantilización (commodification)}

La mercancía es, en primer lugar, un objeto exterior, una cosa que, merced a sus propiedades, satisface necesidades humanas del tipo que sean. (Karl Marx, 1975 [1867]: 43)

El momento central de la neoliberalización de la naturaleza es, probablemente, la mercantilización de ésta última. El neoliberalismo tiene como fetiche el mercado como medio para regular la acción humana. En este sentido, en las sociedades occidentales existe una tendencia creciente a la «mercantilización universal» (universal commodification, ver Radin (1996) o Walsh (1999)) que llega a todos los rincones de la vida moderna (Guthman, 2008; Guthman y DuPuis, 2006; Harvey, 1982; Thrift, 2000; Watts, 1999). Existen unas tentativas continuas para ampliar la mercantilización ${ }^{3}$ de la naturaleza y la circulación de estas nuevas mercancías (Heynen et al., 2007a, 2007b; Liverman, 2004; Lysandrou, 2005).

Sin embargo, al igual que el trabajo o el dinero, el medio ambiente no es inicialmente producido para ser vendido, y entra en la categoría de mercancía ficticia (fictitious commodity, Polanyi (2001 [1944]). En otros términos, Margaret Jane Radin (1996) habla de mercantilización incompleta (incomplete commodification) para referirse a las mercancías que, aún y encontrarse en el mercado, están fuertemente sujetas a la regulación del estado.

Según Castree (2003), sin embargo, no podemos hablar de un solo proceso de mercantilización singular, sino que éste varía en función del sujeto o del objeto que quiere ser mercantilizado. En este sentido, más allá de la cuestión «qué es una mercancía», a la que se habían hecho eco Marx o Polanyi desde perspectivas distintas, Noel Castree se pregunta cuáles son las características que adoptan las cosas cuando se convierten en mercancías. Para este autor, escrutar los procesos de mercantilización sirve para indagar las relaciones entre capitalismo y naturaleza desde una perspectiva aventajada.

Como ya se ha señalado anteriormente, la privatización es el otro proceso clave constituyente del proyecto neoliberal, y muchos autores ven en este proceso un paso clave en la mercantilización del medio ambiente (Castree, 2003; Mansfield, 2008; Prudham, 2008). Castree (2003) destaca otros momentos clave en el proceso de mercantilización: alienación (la capacidad de una mercancía de estar físicamente y moralmente separada de sus vendedores), individuación (la acción de separar una cosa de su contexto, ya sea mediante barreras materiales o legales), abstracción (proceso de homogeneización), valorización y displacement (a través del proceso de desplazamiento, se ocultan las relaciones socioecológicas que sustentan la «producción» de la mercancía).

3. En el presente artículo, utilizo el término «mercantilización» como traducción al castellano de commodification. En algunos textos, se puede leer "comodificación». 


\section{Ambientalismo de mercado y modernización ecológica: paradigmas dominantes en la gestión del medio ambiente a comienzos del siglo XXI}

Una vez presentados los conceptos centrales del binomio formado por neoliberalismo y medio ambiente, es importante ver como la ortodoxia neoliberal se hibridiza con el ambientalismo para promover el uso de mecanismos de mercado en la gestión y la gobernanza ambiental (Mansfield, 2007a; McCarthy y Prudham, 2004). Pero no sólo es interesante ver como el neoliberalismo moldea la gobernanza ambiental, sino como esta última puede ser constitutiva del proyecto neoliberal (Heynen et al., 2007a).

En esta sección, se presentan los dos paradigmas hegemónicos que han dado entrada a los principios neoliberales en la gobernanza ambiental: la modernización ecológica y el ambientalismo de mercado. Desarrollando la idea de Esteban Castro (2009), esta fusión de ecologismo y neoliberalismo pretende desmantelar la gestión pública ambiental y establecer en su lugar un modelo de gobernanza basado en las relaciones sociales capitalistas de propiedad, producción, circulación y acumulación.

\subsection{La modernización ecológica o el sueño tecnocrático del desarrollo sostenible}

En líneas generales, la escuela de la modernización ecológica defiende la compatibilidad entre los objetivos sociales, económicos y ambientales, especialmente a través de la tecnología y del mercado. Este paradigma ambiental, con un componente pragmático muy potente, nació en Alemania bajo el nombre Okologische Modernisierung (Andersen y Massa, 2000; Hajer, 1995) como una crítica a la gestión sumamente burocrática e inefectiva del estado en la década de 1970 (Beck, 1992). Además, resulta la fuente directa de inspiración del famoso informe Brundtland (Brundtland y World Commission on Environment and Development, 1987) y del concepto de desarrollo sostenible. Este paradigma sugiere que no tiene por qué existir una suma igual a cero en el binomio formado por el medio ambiente y el desarrollo económico, sino que existen soluciones que pueden favorecer a ambos.

En este contexto, el rol del estado consiste en implementar nuevos mercados para el desarrollo tecnológico, así como en regular la actividad económica para que ésta sea más sostenible. De acuerdo con este paradigma, las mejoras ambientales no deben ir en detrimento de las lógicas de mercado, sino todo lo contrario: los problemas ambientales se pueden resolver mediante la creación de nuevos mercados, nuevas demandas por parte de la ciudadanía e innovación en la producción y en la organización industrial.

Resumiendo, la modernización ecológica no aboga por un cambio estructural del sistema, sino por soluciones tecnocráticas y apolíticas a los problemas ambientales: eficiencia, gestión basada en criterios técnicos y científicos, innovación tecnológica y producción integrada. Basándose en Hajer (1995), se puede argumentar que la modernización ecológica no solo supuso una respuesta tecnocrática a los problemas ambientales, sino principalmente una estrategia 
de acomodación política de la crítica radical ecologista de la década de 1970 y la internalización y el giro hacia las ideas neoliberales a partir de la década de 1980.

\subsection{El surgimiento del ambientalismo de mercado}

Alimentados por los discursos sobre la modernización ecológica previamente expuestos (Young, 2000), el supuesto fracaso del estado en la gobernanza ambiental (Jänicke, 1990) y los costes crecientes de regulación (Bailey, 2007), los mecanismos de mercado han ganado mucho peso en la gestión ambiental (Andersen y Sprenger, 2000; Jordan et al., 2003; Sprenger, 2000).

El ambientalismo de mercado ${ }^{4}$, de manera análoga a la modernización ecológica, promete la unión entre protección ambiental y crecimiento económico y eficiencia (Anderson y Leal, 1982, 1991, 1997; Meiners y Yandle, 1993). El establecimiento de derechos de propiedad, la utilización de mercados como mecanismos de asignación, la colaboración público-privada y la internalización de las externalidades ambientales a través del precio (Bakker, 2007a, 2007b; Dryzek y Schlosber, 1998; Economy, 2007; Higgins et al., 2010) son los principales mecanismos descritos.

También, de manera análoga a la modernización ecológica, el estado tiene un rol clave en el proceso: el establecimiento de derechos de propiedad sobre el medio ambiente. El ambientalismo de libre mercado asume que los individuos responderán a la información y los incentivos existentes y que el rol del estado es ayudarlos y facilitar este proceso (Pennington, 1999).

Las críticas a este paradigma son varias. Por un lado, Hogg (2000) afirma que existe una sobreestimación de los beneficios de los mecanismos de mercado en la gestión ambiental. Por otro lado, Arild Vatn (2000) argumenta que reducir las cuestiones morales a meras transacciones económicas puede llevar al deterioro de tal moralidad. Más allá de estos argumentos, nos podemos plantear de manera más general si un modelo económico que está en permanente crisis puede guiarnos en la consecución de un medio ambiente más sostenible que pueda ser disfrutado de manera igualitaria y que contribuya a la emancipación individual y colectiva.

\section{Conclusiones}

La agenda neoliberal no se aplica en su forma más pura, sino que el modelo neoliberal existente (Brenner y Theodore, 2002a) surge de la adaptación de los principios neoliberales a contextos específicos donde cohabitan proyectos a priori que no son neoliberales (Castree, 2008b). Las ciudades y las regiones

4. Goldman $(2005,2007)$ no habla de «ambientalismo de libre mercado», sino de «ambientalismo neoliberal» (neoliberal environmentalism) o de "neoliberalismo verde» (green Neoliberalism), si bien se refiere a términos similares. Bernstein (2000) nombra este paradigma como «ambientalismo liberal» (liberal environmentalism). 
tienen un rol clave en las geografías emergentes del neoliberalismo (Brenner y Theodore, 2002b). Sin embargo, es importante también analizar los vínculos multiescalares existentes (Castree, 2005) y las arquitecturas globales en las que se insiere lo local (Peck, 2004). Es aquí donde la geografía parte de una posición aventajada para ayudar a entender cómo se despliegan los procesos de neoliberalización, entre ellos el del medio ambiente.

En un contexto donde el neoliberalismo parece omnipresente (Peck y Tickell, 2002), la geografía crítica tiene la difícil tarea de conectar los debates más abstractos y teóricos (presentados en este artículo) con la investigación empírica. Si no se logra abordar este paso, tendremos, por un lado, narrativas que fracasan en analizar las particularidades contingentes y otras narrativas que se sumen en la especificidad y fracasen en establecer conclusiones generales. Esta tensión entre la necesidad de realizar más estudios de caso empíricos (Larner, 2003) y la de elaborar un marco teórico que nos permita ver las características compartidas y las raíces del proyecto neoliberal (Castree, 2005) tiene que llevar, tal y como argumenta Castree (2008a), a investigaciones que especifiquen las dimensiones neoliberales del caso de estudio. El presente trabajo ha intentado clarificar estas dimensiones por lo que respecta al binomio formado por los conceptos de neoliberalismo y medio ambiente.

El proyecto neoliberal plantea soluciones a los problemas ambientales que se basan en profundizar la hegemonía del mercado y la responsabilidad individual en la gestión ambiental. Más allá de analizar y deconstruir estas tendencias, así como sus consecuencias, la geografía crítica y otras disciplinas, como la ecología política, deben plantear alternativas válidas para alcanzar una sociedad ecológicamente más sostenible y socialmente más justa, que escape de la dinámica permanente de crisis económica y ecológica.

\section{Agradecimientos}

Hug March es investigador postdoctoral Juan de la Cierva (convocatoria 2011), programa financiado por el Ministerio de Economía y Competitividad. El autor quiere agradecer a David Saurí los comentarios realizados en versiones previas del artículo.

\section{Bibliografía}

Andersen, M.S. y Massa, I. (2000). «Ecological Modernization-Origins, Dilemmas and Future Directions». Journal of Environmental Policy and Planning, 2, 337-345.

Andersen, M.S. y Sprenger, R. (2000). Market-based instruments for environmental management politics and institutions. Cheltenham, UK; Northampton, MA, USA: Edward Elgar.

Anderson, T.L. y Leal, T.L. (1982). «The New Resource Economics: Old Ideas and New Applications». American Journal of Agricultural Economics, 64, 928-934.

- (1991). Free market environmentalism. Pacific Research Institute for Public Policy; Boulder. San Francisco; Oxford: Westview Press. 
- (1997). Enviro-capitalists: doing good while doing well. Lanham, Md.; Oxford: Rowman \& Littlefield Publishers.

BAKKER, K. (2003). «Archipelagos and networks: urbanization and water privatization in the South». The Geographical Journal, 169, 328-341.

- (2005). «Neoliberalizing Nature? Market Environmentalism in Water Supply in England and Wales». Annals of the Association of American Geographers, 95, 542-565.

- (2007a). «Neoliberalizing Nature? Market Environmentalism in Water Supply in England and Wales». En: Heynen, N.; Mccarthy, J.; Prudham, S. y Robbins, P. (eds.). Neoliberal Environments: False promises and unnatural consequences. Londres; Nueva York: Routledge, 101-113.

- (2007b). "The "Commons" versus the "Commodity": Alter-globalization, Antiprivatization and the Human Right to Water in the Global South». Antipode, 39, 430-455.

- (2010). Privatizing Water. Governance Failure and the World's Urban Water Crisis. New York: Cornell University Press.

Bakker, K. y Cameron, D. (2002). Good Governance in Municipal Restructuring of Water and Wastewater Services in Canada. Program on Water Issues, Working Paper \#1. Canadà: Munk Centre for International Studies.

Bailey, I. (2007). "Market Environmentalism, New Environmental Policy Instruments, and Climate Policy in the United Kingdom and Germany». Annals of the Association of American Geographers, 97, 530-550.

BAILEY, I. y MARESH, S. (2009). «Scales and networks of neoliberal climate governance: the regulatory and territorial logics of European Union emissions trading». Transactions of the Institute of British Geographers, 34, 445-461.

Bavo (ed.) (2007). Urban Politics Now: Re-Imagining Democracy in the Neoliberal City. Rotterdam: Netherland Architecture Institute (NAI) Publishers.

Beck, U. (1992). Risk Society: towards a New Modernity. Londres: Sage Publications.

Beeson, M. (2007). "Competing Capitalisms and Neoliberalisms: the Dynamics of, and Limits to, Economic Reform in the Asia-Pacific». En: England, K. y Ward, K. (eds.). Neoliberalization: States, Networks, Peoples. Oxford: Blackwell Publishing, 28-47.

Bernstein, S. (2000). «Ideas, Social Structure and the Compromise of Liberal Environmentalism». European Journal of International Relations, 6, 464-512.

Block, F. (2001 [1944]). «Introduction». En: Polanyi, K. The Great Transformation. Beacon Press, Boston, XVIII-XLI.

Brenner, N. y Theodore, N. (2002a). "Cities and the geographies of "actually existing neoliberalism"». Antipode, 34, 349-379.

- (2002b). "Preface: from the "new localism" to the spaces of Neoliberalism». Antipode, 34, 341-347.

BRIDGE, G. (2007). "Acts of enclosure: claim staking and land conversion in Guyana's gold fields». En: Heynen, N.; Mccarthy, J.; Prudham, S. y Robbins, P. (eds.). Neoliberal Environments: False promises and unnatural consequences. Londres; Nueva York: Routledge, 74-88.

Brundtland, G.H. y World Commission on Environment and Development (1987). Our common future. Ginebra: WCED.

Carrier, J.G. (1997). «Introduction». En: Carrier, J.G. (ed.). Meanings of the Market: the Free Market in Western Culture. Oxford: Berg Publishers.

Carvalho, L.F. y Rodrigues, J. (2006). "On Markets and Morality: Revisiting Fred Hirsch». Review of Social Economy, LXIV, 331-347. 
Castree, N. (2003). "Commodifying what nature?». Progress in Human Geography, 27, 273.

- (2005). "The epistemology of particulars: Human geography, case studies and "context"». Geoforum, 36, 541-544.

- (2006). «From neoliberalism to neoliberalisation: consolations, confusions, and necessary illusions». Environment and Planning A, 38, 1-6.

- (2008a). "Neoliberalising nature: the logics of deregulation and reregulation». Environment and Planning A, 40, 131-152.

- (2008b). «Neoliberalising nature: processes, effects, and evaluations». Environment and Planning A, 40, 153-173.

- (2010). «Neoliberalism and the Biophysical Environment: A Synthesis and Evaluation of the Research». Environment and Society: Advances in Research, 1, 5-45.

Castro, J.E. (2009). «Apuntes sobre el proceso de mercantilización del agua: un examen de la privatización en perspectiva histórica». En: Delclòs, J. (ed.). Agua, un derecho y no una mercancía: Propuestas de la sociedad civil para un modelo público de agua. Barcelona: Icaria, 35-55.

Coates, D. (2000). Models of capitalism: growth and stagnation in the modern era. Cambridge: Polity Press.

Crotty, J. (2000). «Structural Contradictions of the Global Neoliberal Regime». Review of Radical Political Economics, 32, 361-368.

Dryzek, J.S. y Schlosberg, D. (1998). Debating the Earth: the environmental politics reader. Oxford; Nueva York: Oxford University Press.

Economy, E. (2007). «Environmental Governance: the Emerging Economic Dimension». En: Carter, N.T. y Mol, A.P.J. (eds.). Environmental Governance in China. Nueva York: Routledge, 23-41.

England, K. y Ward, K. (2007). Neoliberalization: states, networks, peoples. Malden, MA: Blackwell Publishing.

FINGer, M. y Allouche, J. (2002). Water privatisation: trans-national corporations and the re-regulation of the water industry. Londres: Spon.

Friedman, M. (1962). Capitalism and freedom. Chicago; Londres: University of Chicago Press.

Gamble, A. (2006). «Two Faces of Neo-liberalism». En: Robison, R. (ed.). The Neoliberal Revolution. Forging the Market State. Nueva York: Palgrave Macmillan, 20-35.

Goldman, M. (2005). Imperial Nature: the World Bank and struggles for social justice in the age of globalization. New Haven, Conn.; Londres: Yale University Press.

- (2007). "How "Water for All!" policy became hegemonic: The power of the World Bank and its transnational policy networks». Geoforum, 38, 786-800.

Guthman, J. (2008). «Voluntary Food Labels as Neoliberal Governance». En: MansFIELD, B. (ed.). Privatization: Property and the Remaking of Nature-Society Relations. Oxford: Blackwell Publishing, 64-85.

Guthman, J. y DuPuis, M. (2006). «Embodying neoliberalism: economy, culture, and the politics of fat». Environment and Planning D: Society and Space, 24, 427-448.

Hajer, M.A. (1995). The politics of environmental discourse: Ecological modernization and the policy process. Oxford; Nueva York: Clarendon Press.

Harvey, D. (1982). The Limits to Capital. Oxford: Blackwell.

- (1996). Justice, Nature and the Geography of Difference. Oxford: Blackwell.

- (2003). The New Imperialism. Oxford: Oxford University Press. 
- (2005). A Brief History of Neoliberalism. Oxford; Nueva York: Oxford University Press.

Heynen, N.; McCarthy, J.; Prudham, S. y Robbins, P. (eds.) (2007a). Neoliberal Environments: False promises and unnatural consequences. Londres; Nueva York: Routledge.

Heynen, N.; McCarthy, J.; Prudham, S. y Robbins, P. (2007b). «Introduction: false promises». En: Heynen, N.; McCarthy, J.; Prudham, S. y Robbins, P. (eds.). Neoliberal Environments: False promises and unnatural consequences. Londres; Nueva York: Routledge, 1-21.

Higgins, V.; Dibden, J. y Cocklin, C. (20io). «Market instruments and the neoliberalisation of land management in rural Australia». Geoforum, 43, 377-386.

HogG, D. (2000). «The limitations of economic instruments as stimuli for technical change, technological change and innovation». En: Andersen, M.S. y Sprenger, R. (eds.). Market-based instruments for Environmental Management, Politics and Institutions. Cheltenham, UK; Northampton, MA, USA: Edward Elgard, 175-193.

JäNICKE, M. (1990). State failure: the impotence of politics in industrial society. University Park, PA: Pennsylvania State University Press.

Jessop, B. (2002). «Liberalism, Neoliberalism, and Urban Governance: A State-Theoretical Perspective». Antipode, 34, 452-472.

Jordan, A.; Wurzel, R.K.W. y Zito, A.R. (eds.) (2003). "New» Instruments of Environmental Governance? National Experiences and Prospects. Londres: Routledge.

LARner, W. (2000). «Neo-liberalism: Policy, Ideology, Governmentality». Studies in Political Economy, 63, 5-26.

- (2003). "Neoliberalism?». Environment and Planning D: Society and Space, 21, 509-512.

Larner, W.; Le Heron, R. y Lewis, N. (2007). "Co-constituting “After Neoliberalism”: Political Projects and Globalizing Governmentalities in Aotearoa/New Zeland». En: England, K. y Ward, K. (eds.). Neoliberalization: States, Networks, Peoples. Oxford: Blackwell Publishing, 223-247.

Latour, B. (2005). La science en action: Introduction à la sociologie des sciences. París: La Découverte.

Leitner, H.; Sheppard, E.S.; Sziarto, K. y Maringanti, A. (2007). «Contesting Urban Futures: Decentering Neoliberalism». En: Leitner, H.; Peck, J. y ShepPARD, E.S. (eds.). Contesting Neoliberalism: urban frontiers. Nueva York: The Guilford Press, 1-25.

Liverman, D. (2004). "Who Governs, at What Scale and at What Price? Geography, Environmental Governance, and the Commodification of Nature». Annals of the Association of American Geographers, 94, 734-738.

Lysandrou, P. (2005). "Globalisation as commodification». Cambridge Journal of Economics, 29, 769-797.

Lockie, S. y Higgins, V. (2007). «Roll-out neoliberalism and hybrid practices of regulation in Australian agri-environmental governance». Journal of Rural Studies, 23, $1-11$.

Mansfield, B. (2001). "Thinking through scale: the role of state governance in globalizing North Pacific fisheries». Environment and Planning A, 33, 1807-1827.

- (2004). «Rules of Privatization: Contradictions in Neoliberal Regulation of North Pacific Fisheries». Annals of the Association of American Geographers, 94, 565-584.

- (2007a). «Privatization: Property and the Remaking of Nature-Society Relations Introduction to the Special Issue». Antipode, 39, 393-405. 
- (2007b). "Neoliberalism in the oceans: "rationalization", property rights, and the commons question». En: Heynen, N.; McCarthy, J.; Prudham, S. y Robbins, P. (eds.). Neoliberal Environments: False promises and unnatural consequences. Londres; Nueva York: Routledge, 63-73.

- (2008). «Introduction: Property and the Remaking of Nature-Society Relations». En: Mansfield, B. (ed.). Privatization. Property and the Remaking of Nature-Society Relations. Oxford: Blackwell Publishing, 1-13.

Marx, K. (1975 [1867]). El Capital. Tomo I, vol. 2: El proceso de producción del capital. Madrid; Buenos Aires; Ciudad de Méjico: Siglo XXI.

McCarthy, J. y Prudham, S. (2004). «Neoliberal nature and the nature of neoliberalism». Geoforum, 35, 275-283.

Meiners, R.E. y Yandle, B. (1993). Taking the Environment Seriously. Lanham, US: Rowman \& Littlefield Publishers.

Miller, P. y Rose, N. (2008). Governing the present. Cambridge: Polity Press.

Moore, L. (2011). «The neoliberal elephant: Exploring the impacts of the trade ban in ivory on the commodification and neoliberalisation of elephants». Geoforum, 42, 51-60.

Nickson, A. y Franceys, R. (2003). Tapping the water market: the challenge of reform in the urban water sector. Basingstoke: Palgrave.

Peck, J. (2004). "Geography and public policy: constructions of neoliberalism». Progress in Human Geography, 28, 392-405.

Peck, J. y Tickell, A. (2002) «Neoliberalizing Space». Antipode, 34, 380-404.

Pennington, M. (1999). «Free market environmentalism and the limits of land use planning». Journal of Environmental Policy and Planning, 1, 43-59.

Perreault, T. y Martin, P. (2005). «Geographies of neoliberalism in Latin America». Environment and Planning A, 37, 191-201.

Polanyi, K. (2001 [1944]). The Great Transformation. Boston: Beacon Press.

Prudham, S. (2008). "The Fictions of Autonomous Invention: Accumulation by Dispossession, Commodification and Life Patents in Canada». En: MANsfield, B. (ed.). Privatization. Property and the Remaking of Nature-Society Relations. Oxford: Blackwell Publishing, 14-37.

Radin, M.J. (1996). Contested Commodities: the Trouble with Trade in Sex, Children, Body Parts and Other Things. Harvard, MA: Harvard University Press.

Robbins, P. y Luginbuhl, A. (2007). "The last enclosure: resisting privatization of wildlife in the western United States». En: Heynen, N.; McCarthy, J.; Prudham, S. y Robisns, P. (eds.). Neoliberal Environments: False promises and unnatural consequences. Londres; Nueva York: Routledge, 25-37.

RoberTsOn, M.M. (2007). «The neoliberalization of ecosystem services: wetland mitigation banking and the problem of measurement». En: Heynen, N.; McCarthy, J.; Prudham, S. y Robbins, P. (eds.). Neoliberal Environments: False promises and unnatural consequences. Londres; Nueva York: Routledge, 114-125.

SELF, P. (1993). Government by the market? The politics of public choice. Basingstoke: Macmillan Press.

SMith, L. (2004). «The murky waters of the second wave of neoliberalism: Corporatization as a service delivery model in Cape Town». Geoforum, 35, 375-393.

Sмiтн, N. (1984). Uneven Development: Nature, capital and the Production of Space. Oxford: Blackwell.

SPRENGER, R. (2000). «Market-based instruments in environmental policies: the lessons experience». En: Andersen, M.S. y Sprenger, R. (eds.). Market-based instru- 
ments for Environmental Management, Politics and Institutions. Cheltenham, UK; Northampton, MA, USA: Edward Elgard, 3-23.

Stiglitz, J.E. (2001 [1944]). «Foreword». En: Polanyi, K. The Great Transformation. Boston: Beacon Press, VII-XVII.

St. Martin, K. (2007). «Enclosure and economic identity in New England fisheries». En: Heynen, N.; McCarthy, J.; Prudham, S. y Robbins, P. (eds.). Neoliberal Environments: False promises and unnatural consequences. Londres; Nueva York: Routledge, 255-268.

Swyngedouw, E. (2005). "Dispossessing H2O: the contested terrain of water privatization». Capitalism Nature Socialism, 16, 81-98.

Thrift, N. (2000). «Commodities». En: Johnston, R.J.; Gregory, D.; Pratt, G. y Watts, M. (eds.). The Dictionary of Human Geography. Oxford: Blackwell, 95-96.

Tickell, A. y Peck, J. (2003). «Making Global Rules: Globalisation or Neoliberalisation?». En: Peck, J. y Yeung, H. (eds.). Remaking the Global Economy: EconomicGeographical Perspectives. Londres: Sage, 163-181.

Toke, D. (2000). Green politics and neo-liberalism. Basingstoke: Macmillan.

VATN, A. (2000). "Efficiency and fairness: the Norwegian experience with agri-environmental taxation». En: Andersen, M.S. y Sprenger, R. (eds.). Market-based instruments for Environmental Management, Politics and Institutions. Cheltenham, UK; Northampton, MA, USA: Edward Elgard, 111-128.

Von Hayek, F.A. (2000 [1944]). Camino de servidumbre. Madrid: Alianza.

Walsh, A. J. (1999). «Review: [Contested Commodities: the Trouble with Trade in Sex, Children, Body Parts and Other Things]». The Philosophical Quarterly, 49, 257-259.

Watts, M. (1999). "Commodities». En: Cloke, P.J.; Crang, P. y Goodwin, M. (eds.). Introducing Human Geographies. Londres: Hodder Arnold, 305-315.

Wolford, W. (2007). "Neoliberalism and the struggle for land in Brazil». En: Heynen, N.; McCarthy, J.; Prudham, S. y Robbins, P. (eds.). Neoliberal Environments: False promises and unnatural consequences. Londres; Nueva York: Routledge, 243-254.

Young, S.C. (2000). The emergence of ecological modernisation: integrating the environment and the economy? Londres; Nueva York: Routledge. 